\title{
ma

\section{Quelles politiques publiques d'appui à l'agroécologie en Amérique latine et dans les Caraibes ?}

\author{
Éric SABOURIN - Jean-François LE COQ - Sandrine FRÉGUIN-GRESH \\ Jacques MARZIN - Muriel BONIN - Maria Mercedes PATROUILLEAU \\ Luis L.VÁZQUEZ - Paulo NIEDERLE
}

L'agroécologie latino-américaine propose de transformer les systèmes agroalimentaires conventionnels. Elle est portée par des mouvements sociaux qui ont su constituer des coalitions conduisant à sa prise en compte par les politiques publiques. Les instruments de ces politiques sont variés et s'insèrent souvent dans des programmes qui appuient également l'agriculture biologique et l'agriculture durable. Or ces deux types d'agriculture, tout en proposant des pratiques plus écologiques, ne remettent pas en cause les fondements du système agroalimentaire conventionnel. La mise en œuvre d'instruments en faveur de l'agroécologie dépend donc des rapports de force instaurés dans chaque pays. Ce Perspective analyse ces politiques et leur influence sur le développement de l'agroécologie dans huit pays : Argentine, Brésil, Chili, Costa Rica, Cuba, Mexique, Nicaragua, Salvador. Ces politiques restent fragiles face aux soutiens massifs à l'agriculture conventionnelle à grande échelle. Leur défi est donc de convaincre plus largement agriculteurs, consommateurs et décideurs autour des questions de santé publique et de sécurité alimentaire.

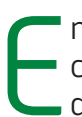

n Amérique latine et dans les Caraïbes, le modèle agricole conventionnel, caractérisé par l'usage intensif d'intrants de synthèse, le travail salarié et la promotion de l'exportation, a longtemps été le seul à être l'objet de politiques publiques. Ce modèle a été remis en cause par les mouvements sociaux : pratiquée dans un cadre peu régulé, l'agriculture conventionnelle a eu des effets néfastes sur la santé publique, l'environnement et l'équité sociale.

Dans ce contexte, d'autres modes de production agricole ont émergé à partir des années 1980. Il s'agit de l'agroécologie (agroecología), de l'agriculture biologique (agricultura orgánica] et de l'agriculture durable [agricultura sostenible] (voir le tableau p. 2). Jusqu'aux années 1990, I'agroécologie était seulement soutenue par les mouvements sociaux, parfois appuyés par la coopération internationale. C'est sous la pression de ces mouvements que certains gouvernements ont commencé à prendre en compte leurs propositions et à répondre partiellement à leurs demandes (voir la figure p. 3).

L'agroécologie latino-américaine s'est faite connaître grâce aux travaux de chercheurs comme Miguel Altieri et Stephen Gliessman, pour être reprise par les mouvements sociaux. Elle propose de transformer radicalement les systèmes agricoles et alimentaires et s'oppose à un modèle conventionnel tourné vers l'exportation, considérant que cette transformation est nécessaire pour relever les défis environnementaux et sociaux.
L'agroécologie partage avec l'agriculture biologique deux principes : produire en préservant les écosystèmes et refuser les intrants non issus de processus biologiques. Mais, en plus, l'agroécologie prône une plus grande autonomie des producteurs vis-à-vis des marchés amont et aval et met en avant le recyclage au sein des écosystèmes agricoles. Elle modifie le système agricole et alimentaire et la relation entre producteurs et consommateurs en proposant les circuits courts et en alliant sécurité et souveraineté alimentaires à l'échelle territoriale. Au-delà de la dimension technique de la production agricole, l'agroécologie offre ainsi une vision intégrale qui conjugue des aspects sociaux, environnementaux, économiques et culturels : elle définit de cette façon un nouveau modèle de développement rural durable.

Les gouvernements à l'écoute des mouvements sociaux, et sensibles aux problèmes que l'agroécologie souhaite résoudre, ont favorisé son émergence en proposant des instruments spécifiques: c'est le cas à Cuba, au Brésil, au Nicaragua et au Salvador.

L'agriculture biologique, promue par l'IFOAM (International Federation of Organic Agriculture Movements) s'est adaptée au modèle économique de l'agriculture conventionnelle. Elle s'en différencie par le refus des intrants de synthèse, qui peuvent être remplacés par des produits naturels. Elle impose des cahiers des charges et exige la certification. Dans les huit 
pays étudiés, l'agriculture biologique est souvent pratiquée par des entrepreneurs familiaux ou des entreprises tournés vers l'exportation, qui voient dans la certification une opportunité commerciale.

L'agriculture durable est plus récente. Elle ne remet pas en cause l'intensification conventionnelle mais y introduit des pratiques agroécologiques associées à des soutiens financiers. L'agriculture durable s'insère dans les mêmes circuits d'approvisionnement et de commercialisation que l'agriculture conventionnelle. Elle est appuyée par des gouvernements libéraux soucieux des enjeux environnementaux, qui veulent protéger les ressources en eau [Mexique, Chili] ou promouvoir une économie verte fondée sur l'écotourisme (Costa Rica). L'agriculture durable privilégie la durabilité environnementale alors que l'agroécologie latino-américaine traite plus largement le pilier social.

\section{Des circonstances qui poussent les politiques vers la promotion de l'agroécologie}

Dans des contextes qui varient selon chaque pays, les gouvernements ont progressivement mis en place des politiques en faveur de l'agroécologie. Dans tous les cas étudiés, trois éléments, de manière indépendante ou simultanée, se retrouvent à l'origine de ces politiques

$>$ des pressions exercées par des mouvements sociaux (incluant des consommateurs). Ces mouvements se mobilisent, se regroupent, parfois au niveau régional, jusqu'à la formulation de politiques. C'est le cas des coalitions qui ont permis l'élaboration de politiques communes à l'agroécologie et à l'agriculture biologique [Brésil, Nicaragua] ;
$>$ la recherche de réponses à des crises: crises économiques et environnementales provoquées par un modèle agroindustriel spécialisé (culture du coton au Nicaragua), crises géopolitiques et financières (effondrement des capacités d'importation d'intrants chimiques à Cuba et au Nicaragua, crise financière en Argentine), évènements climatiques (ouragan Mitch au Nicaragua) ;

$>$ des réponses partielles des pouvoirs publics aux défis régionaux et internationaux : loi du développement rural durable au Mexique (2001) ; reconnaissance de bénéfices environnementaux (paiement pour services environnementaux] appliquée à l'agriculture au Costa Rica (2007) ; plan d'agriculture durable au Chili (2015).

Malgré ces avancées, la production agroécologique reste encore dispersée et limitée. Elle est marginale, sauf à Cuba (65\% des produits agroalimentaires]. Elle est pourtant de plus en plus reconnue comme une des voies possibles pour faire face aux crises qui affectent les systèmes agricoles conventionnels.

Pour développer l'agroécologie, l'accompagnement et le soutien par des politiques publiques sont nécessaires sur deux plans. Premièrement, la complexité des systèmes productifs et la diversité des conditions locales exigent que des références techniques spécifiques soient créées et diffusées localement. Celles-ci doivent être appuyées par des dispositifs d'apprentissage et de gestion des connaissances. Deuxièmement, pour compenser des rendements moindres et des surcoûts de production, notamment en travail, les politiques doivent accompagner la transformation des produits et leur commercialisation. Certains produits peuvent aussi être certifiés via des normes ou des accords entre producteurs et consommateurs (certification participative). Jusqu'à présent,

Amérique latine et Caraïbes : les trois modèles agricoles alternatifs — agroécologie, agriculture biologique, agriculture durable — et le mode de production conventionnel.

\begin{tabular}{|c|c|c|c|c|}
\hline Critères distinctifs & $\begin{array}{l}\text { Agroécologie } \\
\text { latino-américaine }\end{array}$ & $\begin{array}{l}\text { Agriculture } \\
\text { biologique }\end{array}$ & $\begin{array}{l}\text { Agriculture } \\
\text { durable }\end{array}$ & $\begin{array}{l}\text { Agriculture } \\
\text { conventionnelle }\end{array}$ \\
\hline $\begin{array}{l}\text { Type d'exploitation } \\
\text { agricole }\end{array}$ & $\begin{array}{l}\text { Exploitations familiales } \\
\text { (ou paysannes, indigènes] }\end{array}$ & Tous types & Tous types & Tous types \\
\hline Intégration marchande & Limitée & Variable & Maximale & Maximale \\
\hline Système alimentaire visé [1] & Circuits courts & $\begin{array}{l}\text { Circuits longs } \\
\text { et exportation }\end{array}$ & $\begin{array}{l}\text { Circuits longs } \\
\text { et exportation }\end{array}$ & $\begin{array}{l}\text { Circuits longs } \\
\text { et exportation }\end{array}$ \\
\hline $\begin{array}{l}\text { Labellisation de produits } \\
\text { agricoles alimentaires }\end{array}$ & $\begin{array}{l}\text { Possible, plus } \\
\text { ou moins formalisée }\end{array}$ & $\begin{array}{l}\text { Oui, certification } \\
\text { par tiers }\end{array}$ & Non & Non \\
\hline $\begin{array}{l}\text { Échelle de changement } \\
\text { des pratiques agricoles }\end{array}$ & $\begin{array}{l}\text { Parcelle, exploitation, } \\
\text { territoire }\end{array}$ & $\begin{array}{l}\text { Parcelle, } \\
\text { exploitation }\end{array}$ & Parcelle & Parcelle \\
\hline $\begin{array}{l}\text { Intrants [engrais, } \\
\text { produits phytosanitaires...] }\end{array}$ & $\begin{array}{l}\text { Peu d'intrants et issus } \\
\text { de processus biologiques }\end{array}$ & $\begin{array}{l}\text { Issus de processus } \\
\text { biologiques certifiés }\end{array}$ & $\begin{array}{l}\text { Usage raisonné } \\
\text { d'intrants de synthèse }\end{array}$ & $\begin{array}{l}\text { Usage massif } \\
\text { d'intrants de synthèse }\end{array}$ \\
\hline $\begin{array}{l}\text { Culture de variētēs } \\
\text { génétiquement modifiées }\end{array}$ & Non & Non & Oui & Oui \\
\hline $\begin{array}{l}\text { Diversification } \\
\text { des productions dans } \\
\text { l'exploitation agricole }\end{array}$ & Oui & Variable & Non recherchée & Non recherchée \\
\hline $\begin{array}{l}\text { Principaux facteurs } \\
\text { contribuant à la promotion } \\
\text { du modèle }\end{array}$ & $\begin{array}{l}\text { > Politiques publiques } \\
\text { impulsées par les } \\
\text { mouvements sociaux } \\
\text { > Demande des } \\
\text { consommateurs via des } \\
\text { circuits courts ou des } \\
\text { programmes d'achats par } \\
\text { des publics préférentiels }\end{array}$ & $\begin{array}{l}\text { > Demande des } \\
\text { consommateurs } \\
\text { en produits certifiés } \\
\text { biologiques } \\
>\text { Politiques } \\
\text { publiques }\end{array}$ & $\begin{array}{l}\text { > Politiques } \\
\text { publiques incitatives } \\
\text { visant l'adoption } \\
\text { des pratiques } \\
\text { agricoles ciblées }\end{array}$ & $\begin{array}{l}\text { > Politiques agricoles } \\
\text { et commerciales } \\
\text { (accords de libre } \\
\text { commerce) }\end{array}$ \\
\hline
\end{tabular}

(1) : Le système alimentaire a été défini par Louis Malassis : "La façon dont les hommes s'organisent pour produire, distribuer et consommer leur nourriture » (Malassis L., 1994. Nourrir les hommes. Flammarion, Paris, collection Dominos n 16. ISBN 2080351710). 
des efforts de diffusion et de formation ont été réalisés par les mouvements sociaux, les organisations de producteurs, les écoles techniques, les universités, les organisations non gouvernementales et, parfois, les services publics de vulgarisation agricole. Cependant, il manque le renforcement des liens avec les consommateurs pour constituer des coalitions élargies en faveur d'une alimentation saine et respectueuse de l'environnement.

\section{Les instruments des politiques d'appui à l'agroécologie}

Une grande diversité d'instruments politiques concourt à la promotion de l'agroécologie latino-américaine, mais ils sont souvent dispersés entre différents secteurs publics qui ont des difficultés de coordination. On identifie néanmoins quatre types d'instruments, relatifs à l'innovation et au savoir, à l'accès aux ressources, à l'accès aux marchés et à la régulation.

\section{Les instruments de gestion de l'innovation et du savoir ont} trois objectifs : favoriser les échanges horizontaux de savoir et d'expérimentation (programme Campesino a Campesino au Nicaragua et à Cuba], valoriser certaines techniques traditionnelles (Mexique, Chili), promouvoir des réseaux territorialisés de connaissances agroécologiques (programme Ecoforte au Brésil).

Les instruments d'accès aux ressources sont, à l'exemple de Cuba et du Brésil, les actions de réforme agraire et de titularisation foncière, d'accès à l'eau, d'accès au crédit et à la vulgarisation pour les agriculteurs familiaux. Ces actions constituent des bases importantes pour permettre la négociation de programmes plus spécifiques de promotion de l'agroécologie.
Les instruments d'accès aux marchés et de sécurité alimentaire comprennent une large gamme d'actions. Les huit pays étudiés ont des normes de certification biologique, répondant surtout aux exigences des pays importateurs. La certification biologique participative existe au Costa Rica, au Chili, et au Brésil (où fonctionne aussi la certification par contrôle social gérée par des organisations de producteurs). Elle émerge également à Cuba. Les instruments d'appui aux circuits courts sont fondés sur la construction sociale de marchés de proximité : foires, paniers paysans, coopératives de consommateurs, groupes d'agriculture communautaire en ville. D'autres formes de circuits courts ont été diffusés dans toute l'Amérique latine : il s'agit des achats publics préférentiels aux agriculteurs familiaux avec un prix majoré pour les produits agroécologiques ou biologiques, comme les programmes d'acquisition d'aliments au Brésil et d'alimentation scolaire au Nicaragua. Enfin, les programmes d'agriculture urbaine et périurbaine [Cuba, programme ProHuerta en Argentine], de sécurité alimentaire et nutritionnelle (Brésil, Mexique) et le programme Buen Vivir [ou Bien vivre: Nicaragua, Salvador] véhiculent un lien fort avec l'agroécologie.

Les instruments de régulation et de subvention environnementales ne concernent pas que l'agroécologie, mais ils promeuvent des pratiques agricoles plus écologiques. II s'agit de la régulation de la biodiversité agricole, des variétés génétiquement modifiées et de l'usage des sols. Des programmes réduisant l'usage des pesticides existent à Cuba et au Costa Rica. Plusieurs pays ont instauré des subventions pour des pratiques agricoles préservant l'environnement: c'est le cas de la reconnaissance du bénéfice environnemental au Costa Rica, de la protection des eaux et de la biodiversité au Mexique et de l'efficience énergétique au Chili.
Chronologie des actions en faveur des agricultures alternatives en Amérique latine et dans les Caraïbes.

1970
$\begin{aligned} & 1972 \text { IFOAM crée } \\ & \text { (International Federation } \\ & \text { of Organic Agriculture } \\ & \text { Movements) }\end{aligned}$

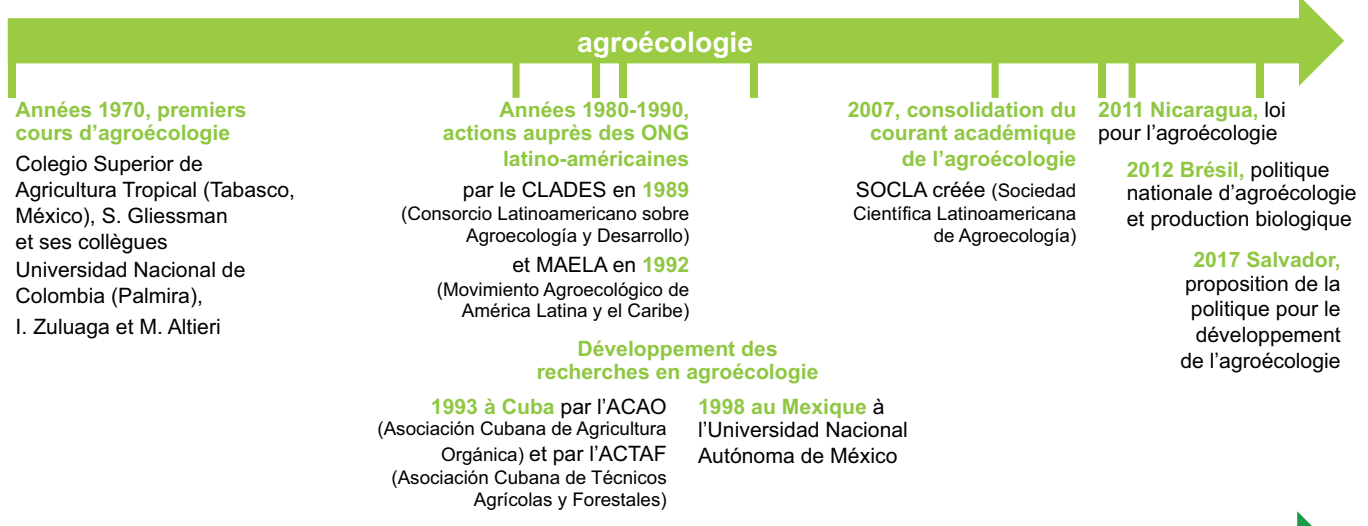

\section{Une mise en œuvre politique active mais complexe}

La lecture historique des politiques latino-américaines montre l'importance du rôle des crises dans l'émergence de l'agroécologie - crises économiques, géopolitiques, environnementales, climatiques. Cette capacité de réponse aux crises fait de l'agroécologie une alternative concrète en termes de durabilité et de résilience face aux défis qui affecteront I'humanité et la planète.

Cela étant, ces politiques doivent surmonter des obstacles de taille. Elles sont en compétition inégale avec les politiques appuyant les acteurs et les firmes de l'agrobusiness. Un important défı est notamment de montrer que la production agroécologique peut fournir des aliments à bas prix pour les plus pauvres, argument par ailleurs revendiqué par l'agrobusiness. Il est également prioritaire de valoriser les apports de l'agroécologie à la santé publique et 
à l'équité sociale, sans se limiter à ses dimensions techniques. Des efforts restent à faire pour mieux coordonner les propositions des mouvements sociaux qui portent l'agroécologie. Celles-ci sont en effet malmenées par le modèle dominant, prétendant être le seul à pouvoir nourrir la planète.

La mise en œuvre et le suivi des politiques en faveur de l'agroécologie dépendent largement de coordinations pour l'instant difficiles à installer entre différents acteurs et niveaux : entre mouvements sociaux portant les modèles alternatifs et institutions agricoles ou organismes publics, entre ministères ou services, entre institutions de régulation des normes, et enfin entre administration nationale et administrations territoriales. L'essor de l'agroécologie latino-américaine sera en particulier lié à des mesures programmées à l'échelle des territoires, alors qu'aujourd'hui elles se limitent en général à celle de la parcelle ou de la ferme. Le territoire correspond à l'échelle de gestion des ressources naturelles, des paysages, des réseaux de connaissances, et de l'organisation du commerce de proximité. Ensuite, le passage de cette échelle locale au niveau national nécessite un ensemble coordonné de politiques spécifiques d'application souple, avec un ancrage social et territorial nourri par des approches participatives. Comme le montrent les exemples au Brésil et à Cuba, les réseaux de production et de partage des connaissances et les circuits courts de vente des produits sont au cœur de cet essor.

Pour convaincre à la fois les producteurs et les décideurs, les instruments politiques promouvant l'agroécologie gagneraient à être flexibles et conçus à plusieurs échelles. L'échelle des territoires est essentielle, pour permettre aux producteurs, aux consommateurs et à leurs organisations d'adapter ces instruments selon leur contexte spécifique.
Ce Perspective $\mathrm{n}^{\circ} 45$ présente les principaux résultats d'une étude des politiques publiques en faveur de l'agroécologie en Amérique latine et dans les Caraïbes, conduite de 2015 à 2017 dans le cadre des travaux du réseau Politiques publiques et développement rural en Amérique latine et Caraïbe (Dispositif de recherche et d'enseignement en partenariat PP-AL, www.pp-al.org/].

L'objectif a été d'analyser les politiques et les instruments qui influencent le développement de l'agroécologie et d'identifier leurs effets et leurs limites à l'échelle nationale et au niveau des territoires locaux. Une grille d'analyse commune, renseignée à partir de données secondaires, d'analyses bibliographiques et d'entretiens, a été utilisée dans huit pays : Argentine, Brésil, Chili, Costa Rica, Cuba, Mexique, Nicaragua, Salvador.
Ces travaux ont aussi donné lieu aux publications suivantes : Fernandes Gabriel B. [Ed.], Romano Jorge O. [Ed.], 2016. Agroecologia e os objetivos do desenvolvimento sustentável. Agriculturas 13 [3]. http://aspta.org.br/revista-agriculturas/.

Rede PP-AL, 2017. Políticas públicas de agroecologia na América Latina e Caribe. AS-PTA (Associação Agricultura Familiar e Agroecologia), Rio de Janeiro, 8 p. http://alianzaagroecologia.redelivre.org.br/2017/05/politicaspublicas-para-a-agroecologia-na-america-latina-e-caribe/.

Sabourin E., Patrouilleau M. M., Le Coq J.-F., Vázquez L., Niederle P.-A. [Eds.], 2017. Políticas Públicas a favor de la Agroecología en América Latina y el Caribe. Porto Alegre, Rede PP-AL, FAO, 412 p. ISBN 978-85-88022-22-5. http://agritrop.cirad.fr/585670/.

\section{Quelques mots sur...}

Éric Sabourin est sociologue au Cirad à I'UMR ART-Dev (Acteurs, ressources et territoires dans le développement, http://art-dev.cnrs.fr/] et professeur visitant de l'Université de Brasilia, Brésil (Universidade de Brasilia, www.unb.br/]. eric.sabourin@cirad.fr

Jean-François Le Coq est agroéconomiste au Cirad à I'UMR ART-Dev et chercheur associé au CIAT à Cali, Colombie (International Center for Tropical Agriculture, http://ciat.cgiar.org/]. II anime le dispositif de recherche et d'enseignement en partenariat PP-AL (Politiques publiques et développement rural en Amérique latine et Caraïbe, www.pp-al.org/] jflecoq@cirad.fr

Sandrine Fréguin-Gresh est agroéconomiste au Cirad à l'UMR ART-Dev à Montpellier, France. Elle intervient au Nicaragua et en Haïti.

\section{sandrine.freguin@cirad.fr}

Jacques Marzin est agroéconomiste au Cirad à l'UMR ART-Dev à Montpellier, France. II intervient à Cuba et en Haïti.

\section{jacques.marzin@cirad.fr}

Muriel Bonin est géographe au Cirad à I'UMR TETIS à Montpellier, France (Territoires, environnement, télédétection et information spatiale, https://tetis.teledetection.fr/index.php/fr/]. Elle a travaillé au Costa Rica. muriel.bonin@cirad.fr

Maria Mercedes Patrouilleau est sociologue, chercheur à I'INTA en Argentine (Instituto Nacional de Tecnología Agropecuaria, https://inta.gob.ar/). Elle travaille sur les politiques publiques.

\section{patrouilleau.mm@inta.gob.ar}

Luis L.Vázquez est agronome et chercheur retraité de l'INISAV à Cuba (Instituto de Investigaciones de Sanidad Vegetal, www.inisav.cu/).

\section{llvazquezmoreno@yahoo.es}

Paulo Niederle est sociologue enseignant-chercheur à l'UFRGS au Brésil [Universidade Federal do Rio Grande do Sul, www.ufrgs.br/ufrgs/inicial]. pauloniederle@gmail.com

\begin{tabular}{|c|c|c|}
\hline (c) & $\begin{array}{l}\text { Cette œuvre est mise } \\
\text { à disposition selon les } \\
\text { termes de la licence }\end{array}$ & $\begin{array}{l}\text { Pour citer ce document } \\
\text { Sabourin E., Le Coq J.-F., Fréguin-Gresh S., Marzin }\end{array}$ \\
\hline $\begin{array}{l}\text { Creative Common } \\
-4.0 \text { Internationa } \\
\text { https://creativec } \\
\text { by/4.0/deed.fr }\end{array}$ & & $\begin{array}{l}\text { Niederle P., 2018. Quelles politiques publiques } \\
\text { d'appui à l'agroécologie en Amérique latine et dans } \\
\text { les Caraïbes? Cirad, Montpellier, Perspective } 45 \text {. } \\
\text { https://doi.org/10.19182/agritrop/00019. }\end{array}$ \\
\hline
\end{tabular}

\section{Quelques liens}

Altieri M. A., 2015. Breve reseña sobre los orígenes y evolución de la Agroecología en América Latina. Agroecología 10 (2): 7-8. www.socla.co/revista/

Altieri M. A., 1995. Agroecology: The Science of Sustainable Agriculture. Second Edition. CRC Press, 448 p. ISBN-13 978-0813317182.

Ecoforte, Programa de Ampliação e Fortalecimento das Redes de Agroecologia e Produção Orgânica (programme d'expansion et de renforcement des réseaux de l'agroécologie et de l'agriculture biologique), Brésil. www.secretariadegoverno.gov.br/iniciativas/brasilagroecologico/ecoforte.

Gliessman S. R., 2014. Agroecology: The Ecology of Sustainable Food Systems. Third Edition. CRC Press, 405 p. ISBN-13 9781439895610.

IFOAM, International Federation of Organic Agriculture Movements. www.ifoam.bio/

Programme ProHuerta, Argentine. http://prohuerta.inta.gov.ar

SOCLA, Sociedad Científica Latinoamericana de Agroecología. Agroecology in Action. http://agroeco.org/.

\section{RED PoĹticas PÚB LICAS

RED POLITICAS PÚBLICAS
Y DESARROLLO RURALEN
AMERICA LATINA www.pp-al.org/

\begin{tabular}{|c|c|}
\hline & $\begin{array}{l}\text { Directeur de la publication : Michel Eddi, } \\
\text { Président directeur général du Cirad }\end{array}$ \\
\hline $\begin{array}{l}\text { CHE ACRONOMIQUE } \\
\text { VELOPPEMENT }\end{array}$ & $\begin{array}{l}\text { Rédacteur.e.s en chef: Patrick Caron, direction générale } \\
\text { déléguée à la recherche et à la stratégie }\end{array}$ \\
\hline $\begin{array}{l}\text { 42, rue Scheffer } \\
75116 \text { Paris } \bullet \text { France }\end{array}$ & $\begin{array}{l}\text { Cécile Fovet-Rabot, délégation à l'information } \\
\text { scientifique et technique }\end{array}$ \\
\hline www.cirad.fr & $\begin{array}{l}\text { Mise en pages et illustrations : Delphine Lavastre-Guard, } \\
\text { délégation à la communication } \\
\text { Diffusion: Christiane Jacquet, délégation } \\
\text { à la communication }\end{array}$ \\
\hline
\end{tabular}

www.cirad.fr/publications-ressources/edition/perspective-policy-brief perspactive ISSN-L 2275-9131 - Courriel : perspective@cirad.fr

\section{A. M U S E}

